\title{
Insulin resistance in man
}

Dear Sir,

Rizza et al. [1] have carried out studies to test the hypothesis of whether or not artificial hyperinsulinaemia may cause or exacerbate insulin resistance in man. The results indicate that short-term, mild hyperinsulinaemia of a magnitude as observed in obesity can decrease insulin-stimulated glucose disposal in man. The lack of change in insulin binding suggests that this insulin resistance had occurred at a post-binding site. Thus, hyperinsulinaemia, whether due to increased secretion or decreased insulin clearance, may be involved in the pathogenesis of insulin resistance in obesity. This conclusion is in accordance with previous studies from our laboratory [2]. We investigated the effect of pharmacologic suppression of insulin secretion by means of diazoxide ( $500 \mathrm{mg}$ /day for four days) on tissue sensitivity in subjects with moderate obesity. Short-term treatment with diazoxide resulted in a decrease of the insulin response to a $100 \mathrm{~g}$ oral glucose challenge by about 30 percent of the initial values. Insulin sensitivity increased by about $50 \%$ in comparison to the pretreatment values. Similarly, there was a marked increase of the antilipolytic effect of insulin. In conclusion, our results and the report of Rizza and co-workers [1] support the concept that insulin resistance in obesity may be secondary to hyperinsulinaemia, which is a characteristic feature of obesity. From the theoretical point of view, a pharmacological sup- pression of insulin secretion may break the vicious circle of hyperinsulinaemia, insulin resistance and glucose intolerance in obesity.

Yours sincerely,

K.P. Ratzmann

\section{References}

1. Rizza RA, Mandarino LJ, Genest J, Baker BA, Gerich JE (1985) Production of insulin resistance by hyperinsulinaemia in man. Diabetologia 28: 70-75

2. Ratzmann KP, Ruhnke R, Kohnert K (1983) Effect of pharmacological suppression of insulin secretion on tissue sensitivity to insulin in subjects with moderate obesity. Int J Obesity 7: 453-458

Dr. Klaus P. Ratzmann

Centre of Diabetes

and Metabolic Disorders

Klosterstr. 71/72

DDR-1020 Berlin 